ORIGINAL RESEARCH

\title{
Patients' Satisfaction with and Preference for Telehealth Visits
}

\author{
Jennifer M. Polinski, ScD, MPH, Tobias Barker, MD, Nancy Gagliano, MD, Andrew Sussman, MD, \\ Troyen A. Brennan, MD, JD, MPH, and William H. Shrank, MD, MSHS \\ CVS Health, Woonsocket, RI, USA.
}

BACKGROUND: One-quarter of U.S. patients do not have a primary care provider or do not have complete access to one. Work and personal responsibilities also compete with finding convenient, accessible care. Telehealth services facilitate patients' access to care, but whether patients are satisfied with telehealth is unclear.

OBJECTIVE: We assessed patients' satisfaction with and preference for telehealth visits in a telehealth program at CVS MinuteClinics.

DESIGN: Cross-sectional patient satisfaction survey.

PARTICIPANTS: Patients were aged $\geq 18$ years, presented at a MinuteClinic offering telehealth in January-September 2014, had symptoms suitable for telehealth consultation, and agreed to a telehealth visit when the on-site practitioner was busy.

MAIN MEASURES: Patients reported their age, gender, and whether they had health insurance and/or a primary care provider. Patients rated their satisfaction with seeing diagnostic images, hearing and seeing the remote practitioner, the assisting on-site nurse's capability, quality of care, convenience, and overall understanding. Patients ranked telehealth visits compared to traditional ones: better (defined as preferring telehealth), just as good (defined as liking telehealth), or worse. Predictors of preferring or liking telehealth were assessed via multivariate logistic regression.

KEY RESULTS: In total, 1734 (54\%) of 3303 patients completed the survey: $70 \%$ were women, and $41 \%$ had no usual place of care. Between 94 and $99 \%$ reported being "very satisfied" with all telehealth attributes. Onethird preferred a telehealth visit to a traditional in-person visit. An additional $57 \%$ liked telehealth. Lack of medical insurance increased the odds of preferring telehealth $(\mathrm{OR}=0.83,95 \% \mathrm{CI}, 0.72-0.97)$. Predictors of liking telehealth were female gender $(\mathrm{OR}=1.68,1.04-2.72)$ and being very satisfied with their overall understanding of telehealth $(\mathrm{OR}=2.76,1.84-4.15)$, quality of care received $(\mathrm{OR}=2.34,1.42-3.87)$, and telehealth's convenience (OR=2.87, 1.09-7.94)

CONCLUSIONS: Patients reported high satisfaction with their telehealth experience. Convenience and perceived quality of care were important to patients, suggesting that telehealth may facilitate access to care.

KEY WORDS: telehealth; patient satisfaction; access to care.

Electronic supplementary material The online version of this article (doi:10.1007/s11606-015-3489-x) contains supplementary material, which is available to authorized users.

Received February 16, 2015

Accepted July 28, 2015

Published online August 13, 2015
J Gen Intern Med 31(3):269-75

DOI: $10.1007 / \mathrm{s} 11606-015-3489-\mathrm{x}$

(C) Society of General Internal Medicine 2015

\section{BACKGROUND}

In the U.S., an estimated $25 \%{ }^{1}$ of patients do not have a primary care provider or do not have complete access to one, particularly in rural areas. ${ }^{2}$ As the U.S. population grows and ages, and millions become insured under the Affordable Care Act, provider resources are increasingly constrained. ${ }^{1}$ In a 2014 survey, $81 \%$ of physicians described themselves as overextended or at full patient capacity, with no time to take on more patients. ${ }^{3}$ Patients find that work and personal responsibilities compete with finding convenient care. ${ }^{4,5}$ In partnerships with many health systems and providers, CVS MinuteClinics offer medical care that supports and augments regular provider care during times when the providers themselves are unavailable. ${ }^{6-8}$ Emerging technologies like telehealth may additionally facilitate access to care when regular providers are not available.

Telehealth enables health care professionals to provide medical services at a distance using video imaging and other technology. ${ }^{9}$ Several studies have found that the quality of telehealth services and patients' clinical outcomes following telehealth visits may be comparable to those of traditional face-to-face office visits, with the additional benefit of superior access to care. ${ }^{10,11}$ Based on the accumulating positive evidence, the Centers for Medicare and Medicaid Services (CMS) has expanded geographic coverage for telemedicine and coverage for new services such as wellness visits and psychotherapy, ${ }^{12-15}$ and 19 states have passed "parity" legislation requiring third-party payers to reimburse for telehealth visits. ${ }^{9}$ The ACA mandates further study of and reimbursement for telehealth services. ${ }^{12,16}$

In October 2013, CVS Health implemented a pilot telehealth program. The program's goals are to provide quality care and to reduce wait times for that care. Patients who visited a pilot clinic and had symptoms appropriate for telehealth consultation could participate in a telehealth visit. Beginning in January 2014, patients were invited to complete a satisfaction survey after their telehealth visits. In this study, we describe 1734 patients' experiences and satisfaction with their telehealth visits. In particular, we explore characteristics and attitudes associated with patients' preference for receiving 
medical care via a telehealth visit compared to a traditional office visit. By understanding the characteristics that improve the patient experience, health care providers can create or improve telehealth programs that provide high-quality, accessible primary care.

\section{METHODS}

\section{The MinuteClinic Telehealth Pilot Program}

Eleven clinics in California and Texas are participating in the program. In the telehealth visit, which mirrors a traditional face-to-face office visit, the patient and an assisting nurse communicate with an off-site nurse practitioner or physician assistant (practitioner) via a video monitor with two-way audio and visual capabilities. The patient describes his/her symptoms, and the assisting nurse uses special diagnostic tools designed for telehealth use (digital video otoscope, telephonic stethoscope, digital video laryngoscope, etc.) to display visual images on the video monitor screen (e.g., the actual images that the otoscope sees when looking in a patient's ear) or present sounds via the monitor's audio output (e.g., the patient's heartbeat detected via the telephonic stethoscope) that the assisting nurse, patient, and remote practitioner can all see or hear. The assisting nurse can also process lab and diagnostic tests. The practitioner provides a diagnostic assessment and, together with the patient, decides upon a treatment plan. The practitioner is able to dispense prescriptions either electronically or via printout by the assisting nurse. The assisting nurse prints out the visit summary and treatment plan. All MinuteClinic providers, whether delivering care on-site or via telehealth, follow the same medical evidence-based treatment algorithms. ${ }^{17}$

\section{Training for the Assisting Nurse}

To participate in the telehealth visit, the assisting nurse participated in 2 weeks of training. During the first week, the assisting nurse completed a series of self-paced online learning modules related to telehealth policies, procedures, and delivery that were developed by MinuteClinic's clinical training managers. During the second week, training managers provided $8 \mathrm{~h}$ ( 1 day) of hands-on training with the telehealth equipment. The assisting nurse then spent the next 4 days practicing with the telehealth equipment in mock telehealth visits together with off-site practitioners and the training managers.

\section{Study Population}

Patients who were 18 years of age or older and who presented at a participating MinuteClinic between January and September 2014 were eligible for a telehealth visit. MinuteClinics are always staffed with an on-site practitioner, and in the pilot program, telehealth visits were available anytime the MinuteClinic was open. All patients who visited a participating MinuteClinic were met by the assisting nurse, who assessed their chief complaints, processed insurance payments, and took vital signs. When the wait to see the on-site practitioner was $20 \mathrm{~min}$ on average, the assisting nurse offered patients with chief complaints appropriate for telehealth consultation (pharyngitis, sinusitis, otitis media, otitis externa, upper respiratory infections, bronchitis, allergic rhinitis, influenza, conjunctivitis, or urinary tract infection) the choice of waiting to see the on-site practitioner or participating in a telehealth visit with a remote practitioner. The assisting nurse informed patients that the cost of the telehealth visit was the same as that for a typical visit. Patients who agreed to participate gave written consent to receive care via telehealth and were placed in a virtual "waiting room." The off-site practitioner initiated the visit by "picking up" the patient from the waiting room. Occasionally, all off-site practitioners were providing telehealth care to other patients, and the on-site practitioner was ready to see the waiting patient before an off-site practitioner. In these cases, the patient saw the onsite practitioner instead. Patients who ultimately participated in a telehealth visit constituted the final study population.

More than half of all patients visiting a MinuteClinic had conditions that were suitable for telehealth consultation. Approximately $70 \%$ of patients offered the telehealth option decided to receive care via telehealth.

\section{Data Sources}

Patient Demographics and Telehealth Satisfaction Survey. Following their telehealth visits, patients were invited to complete a 12-item paper-based survey about their experiences with the MinuteClinic telehealth pilot program team (Appendix available online). The primary purpose for conducting the survey was quality improvement in order to assess whether CVS Minute Clinic's telehealth option satisfied their health care needs. The nursing assistant left the room while patients completed the survey. On the survey, patients were asked whether they had previously received care at a MinuteClinic, had a telehealth visit, and where the telehealth visit(s) took place, and reported their age, gender, whether they had health insurance, and whether they had a PCP. Patients reported their primary motivation for choosing a telehealth visit and any concerns prior to the visit itself. Using a five-point Likert scale ranging from $1=$ very dissatisfied to $5=$ very satisfied, patients rated their satisfaction with each of eight different features: overall understanding of telehealth; ease of seeing diagnostic images on the monitor (e.g., when the digital video otoscope was used, did the patient think that the image he/she saw on the monitor was clear and not blurry); ease of seeing and hearing the remote practitioner; capability of the on-site assisting nurse; quality of the care they received, utility of the treatment and educational materials they received; the convenience of the visit; and overall satisfaction. Patients rated their telehealth visit in comparison to a traditional face-to-face medical visit: better than a traditional visit; just as good as; worse; or not sure. Finally, patients used a four-point Likert scale to indicate how likely they would be to 
use telehealth at MinuteClinic again and how likely they would be to recommend telehealth at MinuteClinic to someone else.

Response Rate. Because the satisfaction survey was conducted to inform program quality improvement activities, no data were collected on the number of patients who were invited to complete a survey (invitations were not made during times when the clinic was out of surveys or when the assisting nurse forgot or was too busy to ask) or on those who refused to participate. All surveys were completed anonymously, and no personal health information or personally identifiable information on survey respondents was collected, in compliance with HIPAA. To estimate the number of surveyeligible patients, we made an ad hoc assumption that $75 \%$ of 4405 telehealth participants during the period January-September 2014 were asked to complete a survey, for an eligible survey population of 3303 . We also report a more conservative response rate that assumes that all 4405 telehealth participants were asked to complete a survey.

Adult MinuteClinic Patient and U.S. Population Demographics. Because no data were collected with which to compare responders with non-responders, we compared responders' demographics with those of adult MinuteClinic patients and the general U.S. adult population. We obtained nationwide age, ${ }^{18}$ gender, ${ }^{19}$ and medical insurance ${ }^{20}$ data from the 2009-2013 American Community Survey conducted by the U.S. Census Bureau. ${ }^{21}$ The proportion of U.S. adults aged 18 years or older with a primary care provider or usual place of care was drawn from the National Health Interview Survey. ${ }^{22}$ We present the raw numbers for each population with no adjustment or standardization.

Outcomes. The main outcome of interest was a patient's preference for telehealth visits compared to traditional office visits. We used patients' responses to the survey question "How did your telehealth visit compare to a traditional inperson visit?" We characterized patients who reported that a telehealth visit was "better than a traditional visit" as "Preferred telehealth" versus all other responses ("just as good as a traditional visit"; "worse than a traditional visit"; or "not sure"). A second outcome, "Liked telehealth," was defined as a patient's rating of a telehealth visit as "better than a traditional visit" or "just as good as a traditional visit" versus all other responses.

Statistical Analyses. In total, 1812 patients completed the survey, of whom 78 reported having used telehealth services prior to the visit of interest. We excluded these 78 patients from further analysis, as repeated telehealth service use would likely bias their responses towards more favorable ratings. The final analytic population included 1734 patients. In conducting the analyses, we used only de-identified data. We described patient demographic characteristics and compared them with the characteristics of the MinuteClinic and U.S. adult populations. Patients' survey responses were calculated for three patient groups: all patients, patients who preferred telehealth, and patients who liked telehealth.

We assessed the relationship between patient demographic and survey responses and their preference for each of the outcomes using univariate regression. We grouped age in three categories (18-34; 35-44; 55 and older) to increase our ability to detect differences across groups. Only survey variables that characterized aspects of patients' experience before or during the telehealth visit were examined, to ensure a temporal association between patient experience and visit preference. These included patients' perceptions of the telehealth visit convenience, concerns with using telehealth, whether they found the telehealth format confusing or complicated, and variables related to their satisfaction with the telehealth process (seeing/hearing the remote practitioner, viewing images, education and materials, treatment plan, capability of assisting nurse, quality of care received, and overall satisfaction). The following variables were excluded: how likely the patient would be 1) to use telehealth again and 2) to recommend telehealth to a friend or family member. Because patients' responses were highly skewed toward the most positive rating, we dichotomized each of the satisfaction variables as "very satisfied" versus "all other" (a grouping of the following responses: "somewhat satisfied," "neither satisfied nor dissatisfied," "somewhat dissatisfied," or "very dissatisfied").

All variables were entered into the corresponding multivariate logistic regression model. All models accounted for the clustering of patients within MinuteClinics using generalized estimating equations. ${ }^{23,24}$ In the multivariate models, we assessed potential collinearity among variables using variance inflation factors (VIF). Analyses were performed using SAS Enterprise Guide 5.1 (SAS Institute Inc., Cary, NC, USA).

\section{RESULTS}

In total, 1734 patients completed a satisfaction survey, for an estimated response rate of $54 \%$. Conservatively assuming that all 4405 telehealth participants were asked to complete a survey (which is not true), the response rate would be $40 \%$. Compared to $61 \%$ of MinuteClinic patients, $70 \%$ of telehealth participants were women (Table 1). Telehealth patients were more likely to report having a PCP or usual place of care $(59 \%)$ than were all MinuteClinic patients (50\%); both were lower than in the U.S. population ( $83 \%)$. Twenty percent of telehealth patients reported not having medical insurance, similar to the prevalence in the U.S. population. In contrast, $11 \%$ of MinuteClinic patients do not have insurance.

One-third (32\%) expressed a preference for receiving care via telehealth. An additional $57 \%$ rated a telehealth visit as "just as good as a traditional visit." One percent of patients 
Table 1 Characteristics of 1734 patients with a telehealth visit, compared with the CVS MinuteClinic general adult population and the U.S. adult population

\begin{tabular}{|c|c|c|c|}
\hline & $\begin{array}{l}\text { MinuteClinic } \\
\text { telehealth } \\
\text { patients } \\
N=1734\end{array}$ & $\begin{array}{l}\text { MinuteClinic } \\
\text { general adult } \\
\text { population }\end{array}$ & $\begin{array}{l}\text { U.S. adult } \\
\text { population } \\
\text { aged } 18 \\
\text { or older } \\
N=237,659,116\end{array}$ \\
\hline & \multicolumn{3}{|l|}{ Percent $(\%)$} \\
\hline Age (years)* & & & \\
\hline $\begin{array}{l}18-24 \\
25-34\end{array}$ & 48 & 40 & 29 \\
\hline $\begin{array}{l}35-44 \\
45-54\end{array}$ & 39 & 36 & 36 \\
\hline $\begin{array}{l}45-54 \\
55-64 \\
65 \text { and older }\end{array}$ & 11 & 21 & 34 \\
\hline Female gender & 70 & 61 & 51 \\
\hline Do not have medical insurance & 20 & 11 & 22 \\
\hline Have a primary care provider ( $\mathrm{PCP})$ or usual place of care & 59 & 50 & 83 \\
\hline First MinuteClinic visit & 50 & - & - \\
\hline
\end{tabular}

* Categories may not sum to 100 due to rounding

rated the telehealth visit as "worse than a traditional visit," and the remainder responded that they were not sure.

Over half of patients cited shorter/no wait time as their primary motivation for using telehealth (Table 2). Patients' experiences were overwhelmingly positive: 94-99\% reported being "very satisfied" with all attributes. When rating the quality of care they received, $95 \%$ of all patients were very satisfied, $98 \%$ rated the assisting nurse as highly capable, and $94 \%$ were very satisfied with the treatment plan and educational materials they received. Among all patients, $95 \%$ were

Table 2 Patients' motivations for and satisfaction with telehealth visits, among all patients and among those who rated telehealth as good or better than a traditional office visit

\begin{tabular}{|c|c|c|c|}
\hline & $\begin{array}{l}\text { All patients } \\
N=1734\end{array}$ & $\begin{array}{l}\text { Preferred telehealth: } \\
\text { "Telehealth visit is } \\
\text { better than traditional } \\
\text { visit" } \\
N=551\end{array}$ & $\begin{array}{l}\text { Liked telehealth: "Telehealth } \\
\text { visit is as good or } \\
\text { better than a } \\
\text { traditional visit" } \\
N=1543\end{array}$ \\
\hline & $\mathrm{N}(\%)$ & & \\
\hline \multicolumn{4}{|l|}{ Primary motivation for using telehealth ${ }^{a}$} \\
\hline Shorter or no wait time & $971(56)$ & $297(54)$ & $865(56)$ \\
\hline Curiosity & $393(23)$ & $138(25)$ & $355(23)$ \\
\hline \multicolumn{4}{|l|}{ Concerns } \\
\hline Concerns about using telehealth ${ }^{\mathrm{b}}$ & $66(4)$ & $12(2)$ & $49(3)$ \\
\hline Found telehealth format to be confusing or complicated ${ }^{\mathrm{c}}$ & $14(1)$ & $2(0.4)$ & $7(0.5)$ \\
\hline \multicolumn{4}{|l|}{ Patients reporting that they were "very satisfied" with: ${ }^{\mathrm{d}}$} \\
\hline Overall understanding of the telehealth service & $1620(94)$ & $530(97)$ & $1463(95)$ \\
\hline Ease of seeing diagnostic images on the monitor & $1634(95)$ & $534(97)$ & $1471(96)$ \\
\hline Ease of seeing and hearing the remote practitioner & $1639(95)$ & $536(98)$ & $1476(96)$ \\
\hline Capability of the assisting nurse & $1684(98)$ & $545(99)$ & $1514(99)$ \\
\hline Quality of the health care received & $1638(95)$ & $538(98)$ & $1481(97)$ \\
\hline Treatment plan and patient educational materials received & $1608(94)$ & $530(97)$ & $1455(95)$ \\
\hline Convenience of using the telehealth service & $1638(95)$ & $538(98)$ & $1481(96)$ \\
\hline Overall satisfaction with the telehealth experience & $1631(95)$ & $538(98)$ & $1485(97)$ \\
\hline \multicolumn{4}{|l|}{ Use of telehealth in the future } \\
\hline \multicolumn{4}{|l|}{ Would personally use telehealth again ${ }^{\mathrm{e}}$} \\
\hline Definitely will & $1138(71)$ & $521(95)$ & $1130(73)$ \\
\hline Probably will & $431(27)$ & $28(5)$ & $388(25)$ \\
\hline \multicolumn{4}{|l|}{ Would recommend telehealth to someone else ${ }^{f}$} \\
\hline Definitely will & $1183(73)$ & $522(95)$ & $1,175(76)$ \\
\hline Probably will & $406(25)$ & $29(5)$ & $360(23)$ \\
\hline
\end{tabular}

${ }^{a}$ The survey question was "What was your primary reason for choosing to use telehealth at MinuteClinic?" Response choices were "shorter wait/no wait"; "satisfied with previous telehealth visit"; "curiosity/wanted to see how it works"; "other"

${ }^{b}$ The survey question was "Did you have any concerns about using telehealth," and response choices were "yes" and "no."

"The survey question was "Was there anything about telehealth that you found confusing or complicated," and response choices were "yes" and "no"

${ }^{d}$ The question stem for all of the satisfaction questions was "Using the scale below, please rate your satisfaction with the following:" The five response choices were: $1=$ very dissatisfied, $2=$ somewhat dissatisfied, $3=$ neither satisfied nor dissatisfied, $4=$ somewhat satisfied, and $5=$ very satisfied

eThe question stem was "How likely would you be to use telehealth at MinuteClinic again?" The four response choices were: $1=d e f i n i t e l y$ will, $2=$ probably will, $3=$ probably will not, $4=$ definitely will not

${ }^{f}$ The question stem was "How likely would you be to recommend telehealth at MinuteClinic to someone else?" The four response choices were:

$1=$ definitely will, 2=probably will, $3=$ probably will not, $4=$ definitely will not 
very satisfied with the technology, i.e., their ability to see and hear the practitioner and to see diagnostic images on the monitor. Finally, $95 \%$ of all patients appreciated the convenience of the service. Nearly $100 \%$ of patients in each group reported that they "definitely" or "probably" would use telehealth personally and would recommend telehealth to someone else.

In the multivariate model, those with no medical insurance had $21 \%$ increased odds of preferring a telehealth visit to a traditional visit (odds ratio $[\mathrm{OR}]=1.21 ; 95 \%$ confidence interval [CI], 1.02-1.43) (Table 3). Several variables predicted whether patients liked telehealth. Female patients had 1.75 odds of liking telehealth (95\% CI, 1.05-2.86). Patients also had greater odds if they were very satisfied with their overall understanding of telehealth $(\mathrm{OR}=2.80 ; 95 \% \mathrm{CI}, 1.81-4.32)$, the quality of care they received $(\mathrm{OR}=2.38 ; 1.47-3.87)$, or the convenience of using telehealth $(\mathrm{OR}=2.85 ; 1.06-7.65)$. Conversely, patients who were very satisfied with the capability of the assisting nurse had reduced odds of liking telehealth $(\mathrm{OR}=0.48 ; 95 \% \mathrm{CI}, 0.29-0.80)$.

\section{DISCUSSION}

Among 1734 patients who participated in a MinuteClinic telehealth visit, $95 \%$ were very satisfied with the quality of the health care they received and rated telehealth as better than or just as good as a traditional visit. Only one factor was a significant predictor of patient preference for telehealth: those with no medical insurance had $20 \%$ greater odds of preferring telehealth to a traditional visit. Patients who were very satisfied with their understanding of telehealth, the quality of care they received, and telehealth's convenience had more than 2.3 times the odds of liking telehealth. These results provide insights into patients' motivations for using telehealth and factors associated with their preference for telehealth visits.

Nearly all patients in the telehealth pilot program were very satisfied with the quality of the care they received. Patient perceptions were in line with evidence from studies that found care quality and clinical outcomes following telehealth visits to be comparable or superior to those of traditional ambulatory visits, emergency department visits, or hospitalization. ${ }^{11}$ To date, telehealth programs have been associated with successful management of acute infection, rapid pediatric triage in the emergency department, and the provision of timely highquality care in disciplines such as mental health, primary care/internal medicine, cardiology, and dermatology. ${ }^{25-31}$ Additionally, retail clinic care for common conditions is associated with comparable ${ }^{32}$ or superior quality and outcomes ${ }^{17}$ compared to ambulatory care facilities or emergency departments.

In our study, patients' insurance status and demographics and their motivations for using telehealth reflect current health care access challenges in the U.S. as provider resources are increasingly constrained. ${ }^{1}$ Although $80 \%$ of telehealth participants had medical insurance, $41 \%$ reported that they did not have a PCP or a usual place of care. Fully $95 \%$ were very satisfied with telehealth's convenience. Notably, $70 \%$ of telehealth participants were women, who are often likely to be juggling work, childcare, and other responsibilities that may impede access to convenient medical care. ${ }^{4,5}$ In a recent study of insured patients' use of an exclusively Web-based telehealth model, $21 \%$ of patients had not accessed medical care in the previous year, $67 \%$ were female, and over onethird of telehealth visits occurred on weekends and holidays when traditional care is difficult to access. ${ }^{11}$ Based on our

Table 3 Characteristics associated with patient preference for telehealth visits compared to traditional visits

\begin{tabular}{|c|c|c|}
\hline & $\begin{array}{l}\text { Preferred telehealth: } \\
\text { "Telehealth visit is better } \\
\text { than a traditional visit" }\end{array}$ & $\begin{array}{l}\text { Liked telehealth: "Telehealth } \\
\text { visit is as good as or better } \\
\text { than a traditional visit" }\end{array}$ \\
\hline & Odds ratio $(95 \% \mathrm{CI})^{\mathrm{d}}$ & \\
\hline Age $18-34$ years & $0.92(0.79-1.06)$ & $0.92(0.59-1.43)$ \\
\hline Age $35-44$ years & Reference group & Reference group \\
\hline Age 45 years and older & $1.05(0.83-1.33)$ & $0.60(0.26-1.36)$ \\
\hline Female gender & $0.92(0.75-1.11)$ & $1.75(1.05-2.86)$ \\
\hline Have a primary care provider $(\mathrm{PCP})$ & $1.01(0.84-1.21)$ & $1.29(0.89-1.88)$ \\
\hline Do not have medical insurance & $1.21(1.02-1.43)$ & $1.79(0.88-3.70)$ \\
\hline Concerns about using telehealth ${ }^{\mathrm{a}}$ & $0.60(0.33-1.11)$ & $0.45(0.17-1.18)$ \\
\hline Found telehealth format to be confusing or complicated ${ }^{b}$ & $0.59(0.18-1.97)$ & $0.09(0.05-0.19)$ \\
\hline Overall understanding of the telehealth service $\mathrm{c}^{\mathrm{c}}$ & $1.29(0.92-1.80)$ & $2.80(1.81-4.32)$ \\
\hline Ease of seeing diagnostic images on the monitor ${ }^{c}$ & $1.20(0.80-1.80)$ & $1.24(0.26-5.87)$ \\
\hline Ease of seeing and hearing the remote practitioner ${ }^{c}$ & $1.46(0.77-2.76)$ & $1.23(0.31-4.88)$ \\
\hline Capability of the assisting nurse ${ }^{\mathrm{c}}$ & $0.61(0.23-1.61)$ & $0.48(0.29-0.80)$ \\
\hline Quality of the health care received ${ }^{\mathrm{c}}$ & $1.47(0.91-2.36)$ & $2.38(1.47-3.87)$ \\
\hline Treatment plan and patient educational materials received ${ }^{\mathrm{c}}$ & $1.38(0.87-2.20)$ & $2.15(0.79-5.80)$ \\
\hline Convenience of using the telehealth service ${ }^{c}$ & $1.58(0.98-2.54)$ & $2.85(1.06-7.65)$ \\
\hline
\end{tabular}

"The survey question was "Did you have any concerns about using telehealth," and response choices were "yes" and "no"

${ }^{b}$ The survey question was "Was there anything about telehealth that you found confusing or complicated," and response choices were "yes" and "no"

${ }^{c}$ The question stem for all of the satisfaction questions was "Using the scale below, please rate your satisfaction with the following:" The five response

choices were: $1=$ very dissatisfied, $2=$ somewhat dissatisfied, $3=$ neither satisfied nor dissatisfied, $4=$ somewhat satisfied, and $5=$ very satisfied

${ }^{d}$ Results from multivariate logistic regression models 
study, patient characteristics ( $70 \%$ female, $41 \%$ with no usual place of care) and their motivations (56\% reported shorter wait time as their primary reason for using telehealth), MinuteClinic - and the telehealth pilot program in particular - appears to be reaching a segment of the adult population who may have difficulty accessing high-quality, timely care.

Our satisfaction results are in line with recent research that found that improved access to care was associated with patient satisfaction. ${ }^{33}$ Lack of medical insurance was the only significant predictor of a preference for telehealth. Medical insurance is a proxy for access to care, and evidence suggests that both retail clinics and telehealth services can provide quality care at lower costs. ${ }^{34-37}$ Among the $95 \%$ of patients who rated telehealth as just as good as or better than a traditional visit, high satisfaction with the quality of care they received and their understanding of the service were both significant predictors of this rating. Equally important to patients' liking telehealth was convenience. Paradoxically, patients' high satisfaction with the capability of the assisting nurse was inversely associated with liking telehealth. It is possible that patients who developed a strong affinity for the on-site nurse decided that the benefits of this in-person interaction, characteristic of a traditional visit, outweighed the other benefits associated with a telehealth visit, or that they felt that the assisting nurse was capable of performing the diagnostic exam alone, without the remote practitioner's input, not understanding that the nurse was not licensed to practice alone. It is difficult to disentangle which of these explanations, if either, is probable without additional information from patients.

The present study uses survey data from a small nonrandomized telehealth pilot program in which the vast majority of respondents were very satisfied with the quality of health care they received, the convenience of that care, and the logistics and technology associated with the telehealth visit. Because the focus of the quality improvement initiative was to assess patient satisfaction with telehealth, the present study does not compare patients who participated in a telehealth visit with those who declined to participate, as preference for either care delivery model is related to both patient exposure to telehealth and the likelihood that they were satisfied with telehealth, and would bias any analysis. The survey instrument was created by the pilot program evaluation team and was not tested for reliability or validity prior to use. While the high degree of satisfaction with the telehealth pilot program among patients who did decide to try telehealth for the first time is an important finding in and of itself, the homogeneity of patients' responses limited our power to identify predictors of rating a telehealth visit as preferable to or just as good as a traditional visit, and is reflected in the wide confidence intervals. Data on the number of patients who were invited to take the survey and who refused were not available, and we could not assess a precise survey response rate or the comparability of survey responders and non-responders regarding their telehealth experiences. In an effort to address these limitations, we used available telehealth visit metrics to estimate a conservative response rate. Additionally, we compared the demographic characteristics of survey respondents with those of the MinuteClinic and U.S. adult populations, providing context as to the generalizability of our results.

In a pilot program providing care via telehealth, $95 \%$ of all participants who completed a satisfaction survey were highly satisfied with the quality of the care they received, the ease with which telehealth technology was integrated into the visit, and the timeliness and convenience of their care. Fully onethird of patients - and particularly those with no health insurance - preferred a telehealth visit to a traditional visit, and nearly all found telehealth to be just as good as or better than a traditional visit, with quality and convenience driving this ranking. Telehealth may be particularly well-suited to patients who have difficulty accessing timely, convenient care.

Conflict of Interest: CVS Health conducted and funded the study. Each of the authors is an employee of and owns stock in CVS Health.

Corresponding Author: Jennifer M. Polinski, ScD, MPH; CVS Health, 100 Scenic View Drive, \#121130, Woonsocket, RI 02864, USA (e-mail:jennifer.polinski@cuscaremark.com).

\section{REFERENCES}

1. U.S. Department of Health and Human Services, National Center for Health Workforce Analysis, . Projecting the Supply and Demand for Primary Care Practitioners Through 2020. Rockville, MD: U.S. Department of Health and Human Services; 2013.

2. Health professional shortage areas. 2014. (Accessed 2015 July 24, at datawarehouse.hrsa.gov/topics/shortageareas.aspx.)

3. Physicians Foundation. Biennial Physician Survey Report. 2014. (Accessed 2015 July 24, at http://www.physiciansfoundation.org/uploads/default/ 2014_Physicians_Foundation_Biennial_Physician_Survey_Report.pdf)

4. Kullgren JT, McLaughlin CG, Mitra N, Armstrong K. Nonfinancial barriers and access to care for U.S. adults. Health Serv Res. 2012;47:462-85.

5. Salganicoff A, Ranji U, Beamesderfer A, Kurani N. Women and health care in the early years of the Affordable Care Act. 2014. (Accessed 2015 July 24, at www.kff.org/womens-health-policy/report/women-and-healthcare-in-the-early-years-of-the-aca-key-findings-from-the-2013-kaiserwomens-health-survey/).

6. CVS Health announces new clinical affiliations with leading health care organizations in Louisiana and Mississippi. 2015. (Accessed 2015 July 24 at http://www.cvshealth.com/newsroom/press-releases/corporate-info-events-cvsminuteclinic-cvspharmacy-minuteclinic/cvs-health-0.)

7. CVS Health announces new clinical affiliations with leading regional health systems. 2014. (Accessed 2015 July 24, at http://www.cvshealth.com/ newsroom/press-releases/cvsminuteclinic-cvspharmacy/cvs-health-announces-new-clinical-affiliations.)

8. Palermo S. CVS partners with Dartmouth-Hitchcock for Minute Clinics in N.H. Concord Monitor 2013: 4 Dec 2013. (Accessed 2015 July 24, at http://www.concordmonitor.com/community/town-by-town/concord/ 9616595-95/cvs-partners-with-dartmouth-hitchcock-for-minute-clinicsin-nh).

9. Weinstein RS, Lopez AM, Joseph BA, et al. Telemedicine, telehealth, and mobile applications that work: opportunities and barriers. Am J Med. 2014; 127:183-7.

10. Nesbitt TS, Marcin JP, Daschbach MM, Cole SL. Perceptions of local health care quality in 7 rural communities with telemedicine. J Rural Health. 2005;2 1:79-85.

11. Uscher-Pines L, Mehrotra A. Analysis of Teladoc use seems to indicate expanded access to care for patients. Health Aff. 2014;33:258-64.

12. Telehealth services. 2014. (Accessed 2015 July 24, at http://www.cms. gov/outreach-and-education/medicare-learning-network-mln / 
mlnproducts/downloads/telehealthservesfctsht.pdf and http://www.cms. gov/Outreach-and-Education/Medicare-Learning-Network-MLN/ MLNProducts/MLN-Publications-Items/CMS1243327.html.)

13. CY 2015 revisions to payment policies under the physician fee schedule and other revisions to Medicare Part B. Regulation \#CMS-1612-FC. 2014. (Accessed 2015 July 24, at http://www.cms.gov/Medicare/Medicare-Feefor-Service-Payment/PhysicianFeeSched/PFS-Federal-Regulation-NoticesItems/CMS-1612-FC.html.)

14. CMS boosts telehealth in 2015 physician pay schedule. 2014. (Accessed 2015 July 24, at http://www.mhealthnews.com/news/cms-booststelehealth-2015-physician-pay-schedule.)

15. Telehealth and Medicare. 2014. (Accessed 2015 Jun 25, at cchpca.org/ telehealth-and-medicare.)

16. Telemedicine in the Patient Protection and Affordable Care Act. 2010. (Accessed 2015 July 24, at americantelemed.org/docs/default-source/ policy/telehealth-provisions-within-the-patient-protection-and-affordablecare-act.pdf.)

17. Shrank WH, Krumme AA, Tong AY, et al. Quality of care at retail clinics for 3 common conditions. Am J Manag Care. 2014;20:794-801.

18. American Community Survey 2009-2013. 2013. (Accessed 2015 July 24 at http://factfinder2.census.gov/faces/tableservices/jsf/pages/ productview.xhtml?pid=ACS_13_5YR_DP05\&prodType=table.)

19. American Community Survey, 2009-2013. 2013. (Accessed 2015 July 24 at http://factfinder2.census.gov/faces/tableservices/jsf/pages/ productview.xhtml?pid=ACS_13_5YR_S0101\&prodType=table.)

20. American Community Survey 2009-2013 5 year estimates. 2013 (Accessed 2015 July 24, at

21. http://factfinder2.census.gov/faces/tableservices/jsf/pages/productview. xhtml?pid=ACS_13_5YR_S2701\&prodType=table.)

22. American Community Survey. 2013. (Accessed 2015 July 24, at http:// www.census.gov/acs/www/data_documentation/2013_release/.)

23. Centers for Disease Control and Prevention. Summary health statistics for US adults: National Health Interview Survey, 2012. (Accessed 2015 July 24, at http://www.cdc.gov/nchs/data/series/sr_10/sr10_260.pdf)

24. Liang KY, Zeger SL. Longitudinal data analysis using generalized linear models. Biometrika. 1986;73:13-22.
25. Liang KY, Zeger SL. Regression analysis for correlated data. Annu Rev Public Health. 1993;14:43-68.

26. Marconi GP, Chang T, Pham PK, Grajower BA, Nagar AL. Traditional nurse triage vs physician telepresence in a pediatric ED. Am J Emerg Med. 2014;32:325-9.

27. Mann SM, Welk TA. Hospice incorporated: combining high tech and high touch care. Kans Nurse. 1997;72:3-4.

28. Aucar JA, Doarn CR, Sargsyan A, Samuelson DA, Odonnell MJ, DeBakey ME. Use of the Internet for long-term clinical follow-up. Telemed J. 1998;4:371-4.

29. Rissam HS, Kishore S, Bhatia ML, Trehan N. Trans-telephonic electrocardiographic monitoring (TTEM) - first Indian experience. Stud Health Technol Inform. 1998;50:361-3.

30. Forkner ME, Reardon TG, Carson GD. Experimenting with feasibility of telemedicine in Alaska: successes and lessons learned. Telemed J. 1996;2:233-40.

31. Callahan EJ, Hilty DM, Nesbitt TS. Patient satisfaction with telemedicine consultation in primary care: comparison of ratings of medical and mental health applications. Telemed J. 1998;4:363-9.

32. Eron L, King $\mathbf{P}$, Marineau M, Yonehara C. Treating acute infections by telemedicine in the home. Clin Infect Dis. 2004;39:1175-81.

33. Mehrotra A, Liu H, Adams JL. al. e. Comparing costs and quality of care at retail clinics with that of other medical settings for 3 common illnesses. Ann Intern Med. 2009;151:321-8.

34. Improving GP services in England: exploring the association between quality of care and the experience of patients. 2012. (Accessed 2015 July 24, at http://www.kingsfund.org.uk/publications/improving-gp-services-england.)

35. Thygeson M, VanVorst KA, Maciosek MV, Solberg L. Use and costs of care in retail clinics versus traditional care sites. Health Aff. 2008;27:128392.

36. Sussman A, Dunham L, Snower K. al. e. Retail clinic utilization associated with lower total cost of care. Am J Manag Care. 2013;19:e148-e157.

37. Baker LC, Johnson SJ, Macaulay D, Birnbaum H. Integrated telehealth and care management program for medicare beneficiaries with chronic disease linked to savings. Health Aff. 2011;30:1689-97. 